\title{
The Atlas Muon Trigger Performance: Run 1 and Initial Run 2 Performance
}

\author{
Kota Kasahara* on behalf of the ATLAS Collaboration \\ University of Tsukuba (JP) \\ E-mail: kota.kasahara@cern.ch
}

\begin{abstract}
Events with muons in the final state are an important signature for many physics topics at the Large Hadron Collider. An efficient trigger on muons and a detailed understanding of its performance are required. In 2012, the last year of Run 1, the instantaneous luminosity of the LHC reached $7.7 \times 10^{33} \mathrm{~cm}^{-2} \mathrm{~s}^{-1}$ and the average number of events that occur in a same bunch crossing was 25 . The ATLAS Muon trigger has successfully adapted to this changing environment by making use of isolation requirements, combined trigger signatures with electron and jet trigger objects, and by using so-called full-scan triggers, which make use of the full event information to search for di-lepton signatures, seeded by single lepton objects. A stable and highly efficient muon trigger was vital in the discovery of Higgs boson in 2012 and for many searches for new physics. The performance of muon triggers during the LHC Run 1 data-taking campaigns is presented, together with an overview and preliminary results of the new muon strategy and algorithms, designed to face the demanding and challenging environment, which will be adopted during Run 2.
\end{abstract}

The European Physical Society Conference on High Energy Physics 22-29 July 2015

Vienna, Austria

\footnotetext{
* Speaker.
} 


\section{Introduction}

The Large Hadron Collider (LHC) is a proton-proton collider for elementary particle physics research at the world highest energies, located at the European Organization for Nuclear Research (CERN) in Switzerland. The center-of-mass energy of the collisions was 7 and $8 \mathrm{TeV}$ during 2011 and 2012 respectively (Run 1). The accelerator went through an upgrade since, and Run 2 started in 2015. The center-of-mass energy has been increased to $13 \mathrm{TeV}$.

The collision rate of the LHC was $20 \mathrm{MHz}$ in Run 1 and at the beginning of Run 2. It was raised to the design value of $40 \mathrm{MHz}$ in 2015 when bunch space was changed to $25 \mathrm{~ns}$ from 50 ns. The size of data from the ATLAS detector [1] at a collision point of the LHC is about 1.5 MB for each collision, amounting to a data rate of $60 \mathrm{~TB} / s$. It is not realistic to record all data taken during collisions on permanent storage. Therefore the ATLAS experiment has a trigger system in place to select the most interesting events so that the event rate being written to tape data storage is reduced to about $1 \mathrm{kHz}$. Maintaining adequate performance of the trigger system is essential, yet challenging for the success of the physics programs of the experiment, and the high energy collisions of the LHC at the high rate makes such effort very challenging.

Events containing muons in the final state offer clean datasets to study interesting physics and triggering on muons is especially important in high energy collider experiments. For example, the discovery of the Higgs boson in 2012 [2] was significantly driven by the search for $H \rightarrow Z Z^{*}$ decays at the ATLAS experiment, where half of the analysis sample was triggered with muons. In this note, we briefly summarize the performance of the muon trigger in the ATLAS experiment in Run 1, implemented to confront the challenges of the LHC environment in Run 2.

\section{Trigger and data acquisition systems and the muon spectrometer}

The ATLAS trigger system was a three-tiered system in Run 1 and is shown in Figure 1. Level 1 (L1) was a hardware based trigger, selecting interesting events using track and energy information constructed in dedicated fast trigger systems. The L1 trigger had a maximum accept rate of $70 \mathrm{kHz}$ within a latency of $2.5 \mu \mathrm{s}$, and defines Regions-of-Interest (RoI) [3] as the geographical location of muon candidate. Then, the objects were reconstructed at the Level 2 (L2) combining more detailed information only available in the RoI. Thus, the output rate at L2 was $6.5 \mathrm{kHz}$, the average processing time $40 \mathrm{~ms}$. Finally, the trigger objects are reconstructed using full event information at the Event Filter (EF), where precise offline-like algorithms are applied. The EF has an output rate of $1 \mathrm{kHz}$ and an average processing time of $4 \mathrm{~s}$.

The L2 and EF selections were executed on two separate computer farms in Run 1. In Run 2, they have been merged into a single High-Level Trigger (HLT) farm. The logic of the HLT underlying the trigger decision is similar to the case of Run 1. 


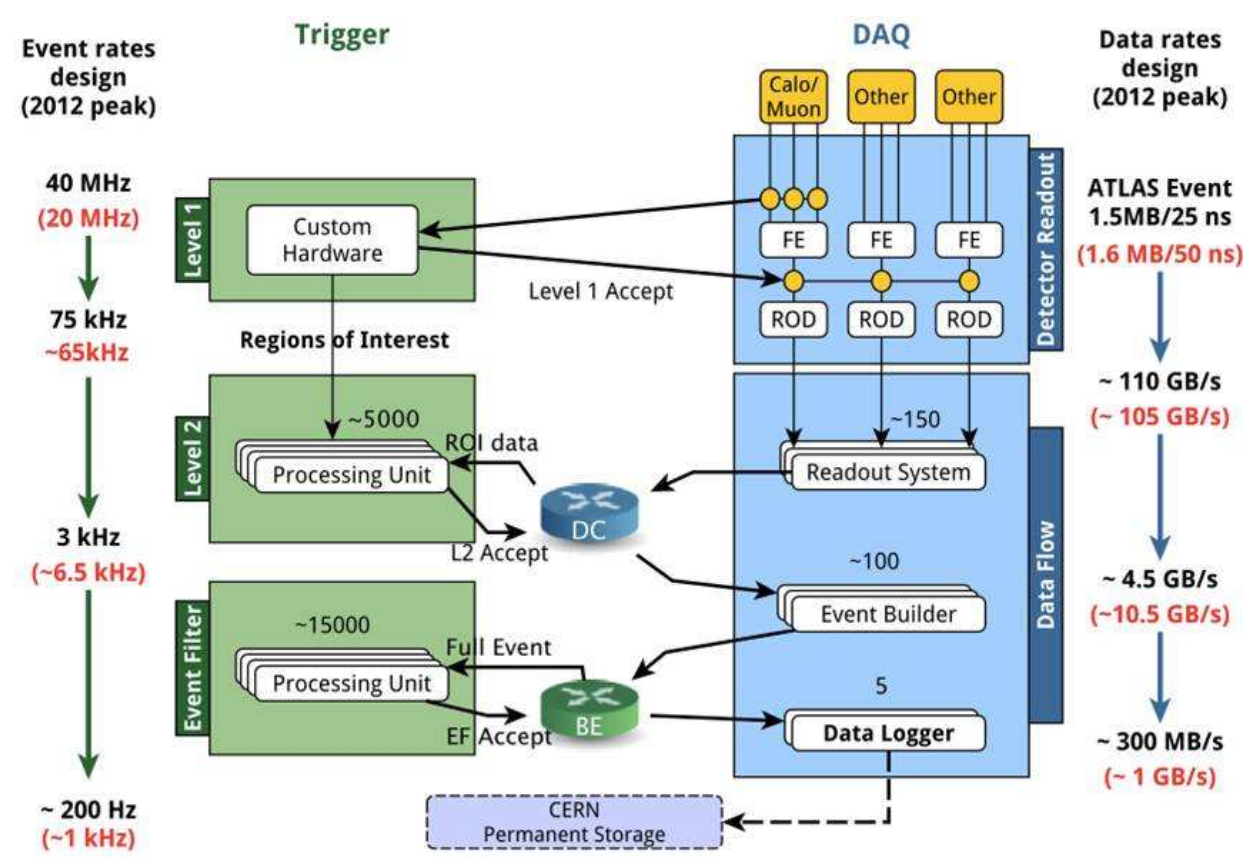

Figure 1: Outline of the trigger and the data acquisition systems in Run 1. The three levels of trigger decisions is shown on the left side, and the flow of data in the data acquisition is on the right side. The design values and the 2012 peak values for the event and data rates are shown in black and red respectively. The Level 2 and EF are merged into one system (HLT) in Run 2.

The muon spectrometer is composed of three large air-core superconducting toroidal magnet systems, one in the central region and two in the endcap and four kinds of detectors, which cover a large solid angle around collision point. Each toroidal magnet system is constructed from eight separate superconducting coils. A schematic view of the muon system is shown in Figure 2.

The Resistive Plate Chamber (RPC) and the Thin Gap Chamber (TGC) have fast response (nanoseconds), which generate the L1 muon trigger

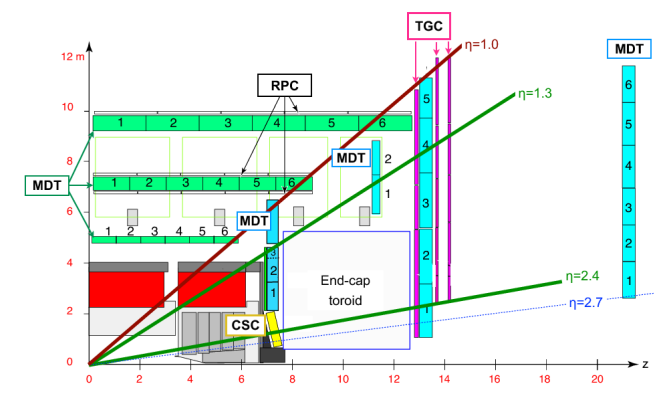

Figure 2: A schematic view showing a quarter-section of the muon spectrometer in a plane containing the beam axis. Taken from Ref. [4]. by checking the spatial and temporal coincidence of hit information. In the barrel region $\left(\left|\eta^{1}\right|<1.05\right)$, the L1 trigger generated by RPCs requires a coincidence of hits in the three layers. In the end-cap region $(1.05<|\eta|<2.4)$, the L1 trigger generated by TGCs hits information checks a coincidence

\footnotetext{
${ }^{1}$ ATLAS uses a right-handed coordinate system with its origin at the nominal interaction point (IP) in the centre of the detector and the $z$-axis along the beam pipe. The $x$-axis points from the IP to the centre of the LHC ring, and the $y$-axis points upward. Cylindrical coordinates $(r, \phi)$ are used in the transverse plane, $\phi$ being the azimuthal angle around the beam pipe. The pseudorapidity is defined in terms of the polar angle $\theta$ as $\eta=-\ln \tan (\theta / 2)$.
} 
of the three layers.

Precision tracking is achieved by the Monitored Drift Tube (MDT) in the region $|\eta|<2.7$ and by the Cathode Strip Chamber $(C S C)$ in the region $2<|\eta|<2.7$.

\section{Muon trigger performance in Run 1}

The tag-and-probe method is used to measure the trigger efficiency using di-muon events. If one of the muons passed the trigger to record the event ("tag"), then the other muon ("probe") is used to estimate the efficiency by examining the probability for it to also pass the trigger. Events with a pair of muons from $Z$ or $J / \psi$ decays reconstructed in the offline analysis are used. The tagand-probe method requires that the invariant mass of the muon pair is consistent with a $Z$-boson or $J / \psi$ meson. The $Z$-boson events covers a wide range of muon $p_{\mathrm{T}}, 10 \mathrm{GeV} \leq p_{\mathrm{T}} \leq 100 \mathrm{GeV}$, and the $J / \psi$ meson events cover a lower muon $p_{\mathrm{T}}$ region, $p_{\mathrm{T}} \leq 10 \mathrm{GeV}$.

Figure 3 shows the efficiency to pass either the mu24i or the mu36 triggers which served as the main single-muon trigger in many physics analyses in Run 1 [4]. Here the numbers 24 and 36 denote the transverse momentum threshold, where the isolation requirement is denoted as "i", e.g. in mu24i. The data agree very well with the events from Monte Carlo (MC) simulation. Figure 4 shows the efficiency to pass either the mu24i or mu36 trigger as measured individually at each trigger level (L1, L2 and EF). The efficiency curves turn on sharply, the higher the threshold the steeper the turn on curve.

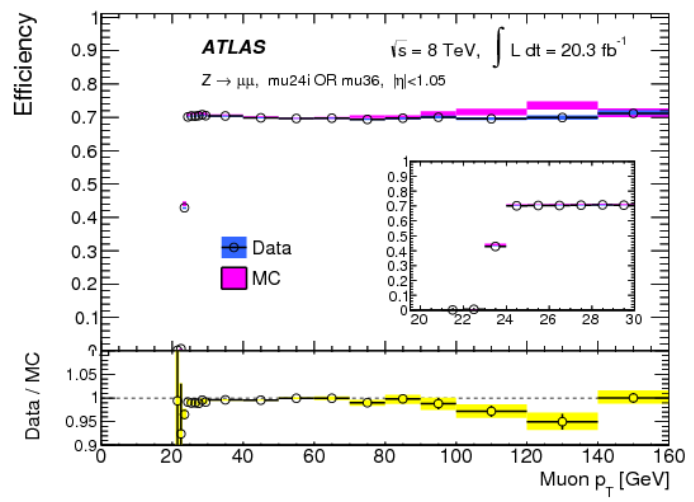

(a)

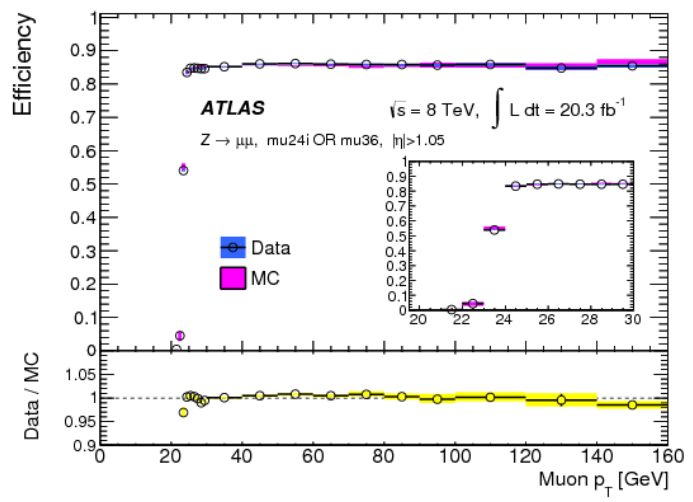

(b)

Figure 3: Efficiency of passing either mu24i or mu36 triggers as function of the probe muon transverse momentum $p_{\mathrm{T}}$ in (a) the barrel region and (b) the endcap region. Efficiencies measured in the data and MC simulation are shown in open circles and in bands, respectively. The ratios of the data divided by MC are shown in the bottom. The error bars include both statistical and systematic uncertainties. Taken from Ref. [4]

\section{Challenges for Run 2 muon triggering}

With the increase of the center-of-mass energy from 8 to $13 \mathrm{GeV}$, and the change in the bunch spacing from 50 to $25 \mathrm{~ns}$ in Run 2, the single lepton trigger rate is expected to increase by a factor 


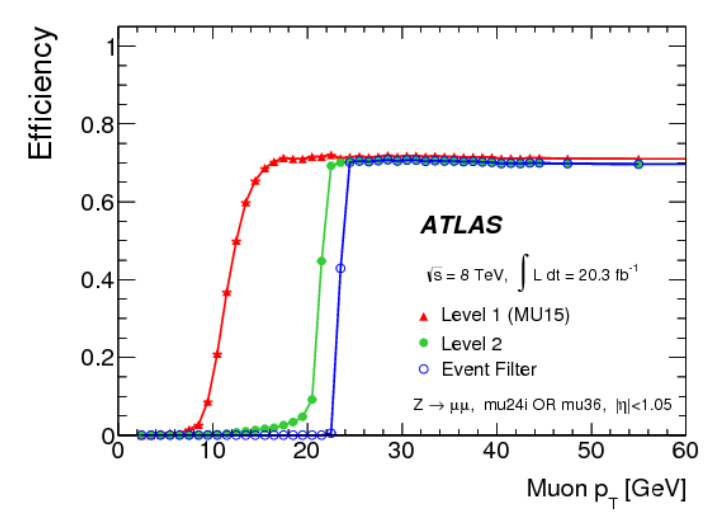

(a)

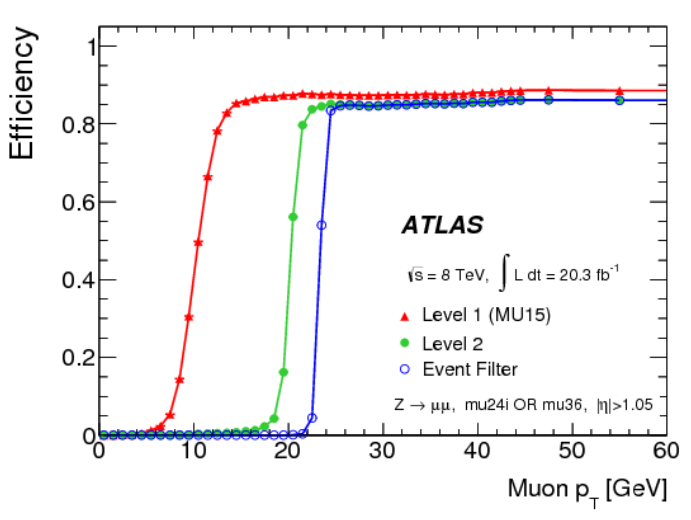

(b)

Figure 4: Efficiency of passing either mu24i or mu36 triggers as function of the probe muon transverse momentum $p_{\mathrm{T}}$ measured at the three trigger levels (L1, L2 and EF) in (a) the barrel region and (b) the endcap region. The error bars show the statistical uncertainties only. Taken from Ref. [4]

of 4 compared to Run 1 . Therefore, the trigger system had to undergo various upgrades which will be described in the following.

\subsection{L1 muon trigger upgrade for Run 2}

The L1 muon trigger rate was suffering from a high fake rate in Run 1. Figure 5 shows the trigger rate of $\mathrm{L} 1$ muons with $p_{\mathrm{T}} \geq 15 \mathrm{GeV}$ as a function of muon $\eta$. Most of the fake muons are found in high $\eta$ regions where the fakes are secondary particles produced in the end-cap material. Such fake triggers can be reduced by introducing the coincidence between the inner TGC (or tile calorimeter signal timing) and outer TGC. Figure 6 shows a schematic view of these detectors ("Tile calo" refers to hadron calorimeter). Figure 7 shows the muon detection efficiency and the L1 trigger rate reduction as a function of tile calorimeter cell energy sum threshold [5]. In the calculation of the energy sum, energies of calorimeter cells (D5 and D6) were smeared by $200 \mathrm{MeV}(1 \sigma)$ in order to emulate the energy resolution measured at L1. For a tile calorimeter energy threshold of $500 \mathrm{MeV}$, the muon detection efficiency is kept as high as $97 \%$ whereas the $\mathrm{L} 1$ rate is reduced by $82 \%$.

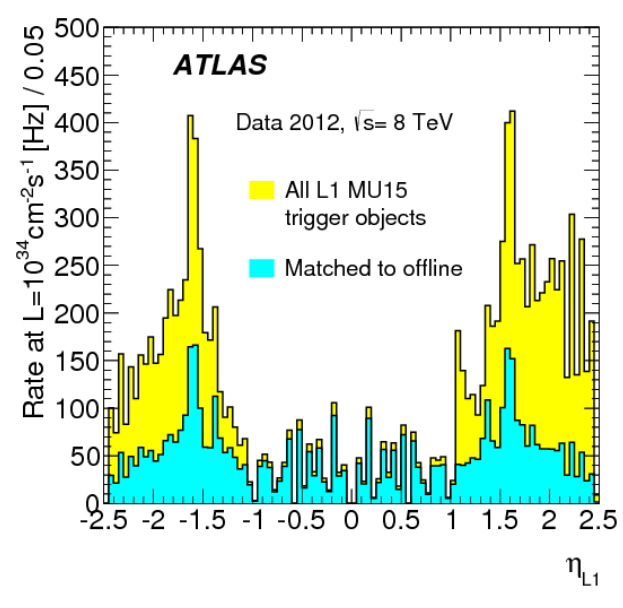

Figure 5: Distributions of L1 muon trigger rates $\left(p_{\mathrm{T}} \geq 15 \mathrm{GeV}\right)$ as function of $\eta$. The yellow hatched histogram shows all muon objects, whereas cyan histogram shows the objects that are identified as muons in the offline reconstruction. Taken from Ref. [4] 


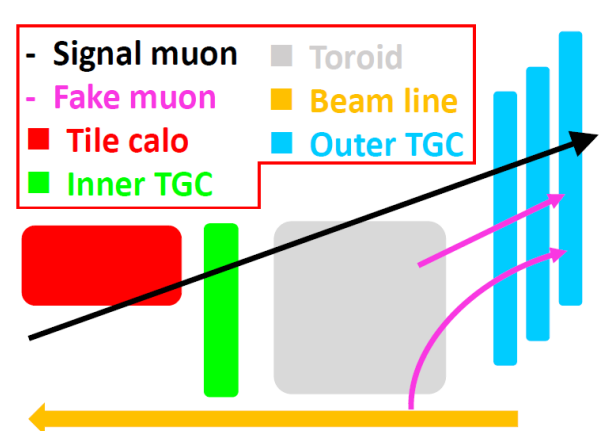

Figure 6: Schematics of the TGCs and tile calo locations within the ATLAS detector. The genuine muons pass through inner detectors, inner TGC or Tile calo, and outer TGC, while fakes are generated around the end-cap material.

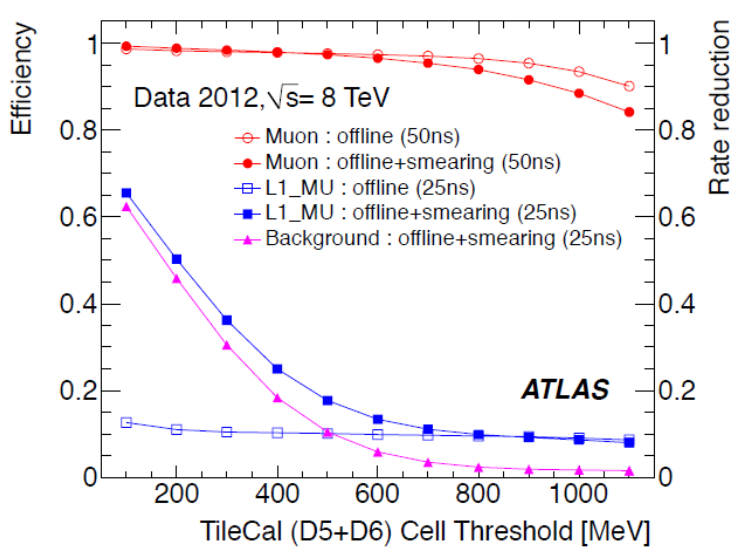

Figure 7: Muon detection efficiency (red) and L1 muon trigger rate reduction (blue) as function of the hadron calorimeter cell energy sum threshold. Taken from Ref. [5]

\section{Conclusion}

The ATLAS muon trigger has been stable and highly efficient in the Run 1 data taking period. The upgrade of the LHC for Run 2 made it necessary to upgrade the trigger system to keep the trigger rate at a sustainable level while still efficiently select events of interest. In Run 1, a significant fake muon rate above $|\eta|$ of 1.05 was observed. The endcap region has been incorporated into the endcap muon trigger of the HLT. Studies show that this additional coincidence will reduce the fake rate by $80 \%$ while maintaining a muon efficiency of almost $100 \%$.

\section{References}

[1] ATLAS Collaboration, The ATLAS Experiment at the CERN Large Hadron Collider, JINST 3 (2008) S08003.

[2] ATLAS Collaboration, Observation of a new particle in the search for the Standard Model Higgs boson with the ATLAS detector at the LHC, Phys. Lett. B 716 (2012) 1, arXiv: 1207 . 7214 [hep-ex] .

[3] R. Blair, J. Dawson, G. Drake, W. Haberichter, J. Schlereth, J. Zhang, M. Abolins, Y. Ermoline, and B. Pope, The ATLAS High Level Trigger Region of Interest Builder, Journal of Instrumentation 3 no. 04, (2008) P04001.

[4] ATLAS Collaboration, Performance of the ATLAS muon trigger in pp collisions at $\sqrt{s}=8 \mathrm{TeV}$, Eur. Phys. J. C 75 (2015) 120, arXiv:1408.3179 [hep-ex] .

[5] R. S. Bartoldus, C. M. C. Bee, D. C. Francis, N. R. Gee, S. L. R. George, R. M. S. Hauser, R. R. Middleton, T. C. Pauly, O. K. Sasaki, D. O. Strom, R. R. I. Vari, and S. R. I. Veneziano, Technical Design Report for the Phase-I Upgrade of the ATLAS TDAQ System, Tech. Rep. CERN-LHCC-2013-018. ATLAS-TDR-023, CERN, Geneva, Sep, 2013. 